\begin{tabular}{|c|c|}
\hline \multirow{3}{*}{ 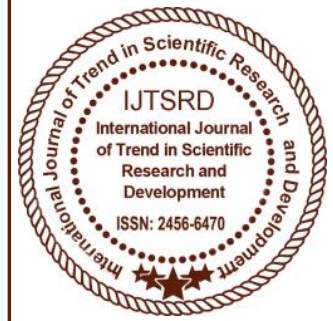 } & $\begin{array}{l}\text { International Journal of Trend in Scientific } \\
\text { Research and Development (IJTSRD) }\end{array}$ \\
\hline & UGC Approved International Open Access Journal \\
\hline & ISSN No: 2456 - 6470 | www.ijtsrd.com | Volume - 1 | Issue - 5 \\
\hline
\end{tabular}

\title{
Advanced Security System using PIC through Bluetooth
}

\author{
G. Naveen Balaji \\ Assistant Professor, Department of \\ ECE, SNS College of Technology, \\ Coimbatore, India
}

\author{
K. Saravanan \\ UG Student, Department of ECE, \\ SNS College of Technology, \\ Coimbatore, India
}

\author{
R. Poorani \\ UG Student, Department of ECE, \\ SNS College of Technology, \\ Coimbatore, India
}

\author{
T. Vishnu Priya \\ UG student, Department of ECE, Coimbatore \\ Institute of Technology, Coimbatore, India
}

\author{
R. Reka Raj \\ UG student, Department of ECE, United \\ Institute of Technology, Coimbatore, India
}

\begin{abstract}
The objective of our project is to develop an Exploiting Bluetooth on Android mobile devices for home appliances control appliances control application. Mobile devices have been integrated into our everyday life consequently, home automation is becoming increasingly prominent features on mobile devices. We have developed a security system that interfaces with an android mobile devices. The mobile device will be communicated through Bluetooth by the shortest way. The mobile application can be loaded onto any compatible device, and once loaded, interface with the appliances easily .commands to $\mathrm{ON}$, OFF, or check the status of the appliance to which is installed can be sent quickly from the mobile device via a simple, easy to use GUI. The security system then acts on these commands, taking the appropriate action and sending a confirmation back to mobile device. This system can also tell the user if any of appliances is $\mathrm{ON}$ or $\mathrm{OFF}$
\end{abstract}

\section{INTRODUCTION}

The objective of our project is to develop an exploiting GSM (Global System for Mobile communication) on android mobile devices for home appliances control application. Mobiles are used in our daily life that used to control the home appliances. Mobile devices have been integrated in to our everyday life .consequently home automation becoming increasingly prominent features on mobile devices.

Our project consists of microcontroller, LCD display, keypad, UART, Bluetooth modem relay, relay driver and android smart phone. The status information is wirelessly transferred to the mobile and the same is displayed at the LCD display. Thus the Bluetooth functionality in the android mobile phone has been effectively leveraged for implementing the home appliances system and as the android phone has been effectively leveraged for implementing the home appliances system and as the android phone is hand held; it is easy to get notifications like lock status etc. At the application itself.

The microcontroller was connected in all blocks of the circuit. The block diagram consists of Transmitter and Receiver. Android smart phone is connected to the transmitter and GSM is connected to the receiver. The components placed in block diagram like micro controller UART (Universal Asynchronous Receiver and Transmitter), GSM (Global System for Mobile Communication), LCD 
(Liquid Crystal Display), relay driver, and power supply.

In our Project we are using 4.0 version android it is "blue tooth". The $12 \mathrm{v}$ electromagnetic relay used. The signal is transmitted from the android smart phone and signal is received by gsm. Gsm 900 is used. By using we can control the devices maximum up to 35 kilometres home appliances.

\subsection{ANDROID}

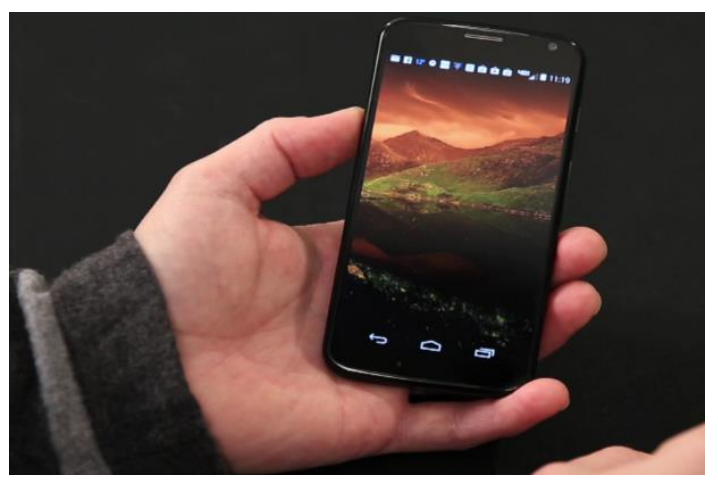

Fig.1 Android Mobile

\subsection{WORKING PRINCIPLE}

Android it is one of the operating system developed in last 15 Years. Android increment was founded in rich miner, nick sears and Chris white in the year 2003. The android has many versions it is also an open mobile phone platform that was developed by Google later by the open handset alliance. Google defines android as 'Software stack'. The software stack made as operating system platform while everything is running thee middleware (the programming allows application to talk to a network and to another )and the application (the actual programs that the phones will run).in short the android software that will make an android phone an android.

\subsection{HOME AUTOMATION}

It is the use it can scheduling and automatic operation of water sprinkling, heating, and air conditioning, window coverings, security systems, lighting ,food preparation etc....home automation may also allow vital home function to be controlled of one or more computers to control basic home functions and features automatically and sometimes remotely. An automation home is sometimes is called a "smart home" remotely from anywhere in the world using a computer connected to the internet. Besides the function already mentioned, remote control can be extended to telephone and answering machines, fax machines and other communication equipment and home robots such as automatic vacuum cleaners.

The fundamental components well designed home automation system include a computers withappropriate programming the various devices and systems to be controlled, interconnecting cables or wireless links a high-speed internet connection, and an emergency backup power source for the computers its peripherals 'and the essential home systems.

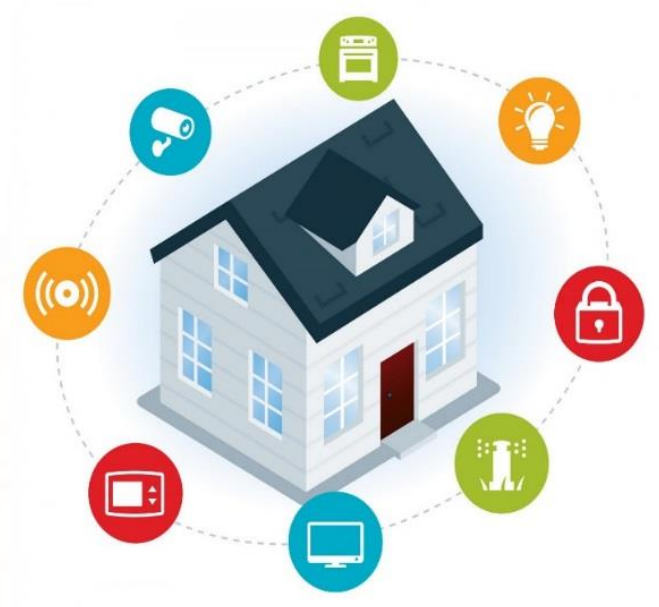

Fig.2 Home Auto Machine

\subsection{PCB (PRINTED CIRCUIT BOARD)}

The printed circuit board (PCB) mechanically supports and electrically connects electronic components wing conductive tracks. Pads other features etched from copper sheets laminated on to a non-conductive substrate. $\mathrm{PCB}$ 'S can be single sided (one copper layer), double side (two copper layer) or multilayer conductors on different layers are connected with plated through holes called visa. Advanced $\mathrm{PCB}^{\text {' } S}$ may contain components like capacitor, resistors or active devices-embedded device in the substrate.

PCB is used in all but the simplest electronic products. Alternatively to the PCB include wire wrap and point to point constriction. PCB'S required additional design effort to layout the circuit. But once that done, the manufacture and assembly can use automation PCB can manufacture circuits less expensively and more quickly than other wiring methods because component mounting and wiring is 
done with one component. Furthermore, operator wiring errors are eliminated

When the board has only copper connections and no embedded components it is more correctly called a "printed wiring board" (PWB) or etched wiring board. Although more accurate the term printed wiring board has fallen in to disuse. A PCB populated with electronic components called a "printed circuit assembly" (PCA)/ (PCBA). The term PCB is used informally both for bare and assembled boards.

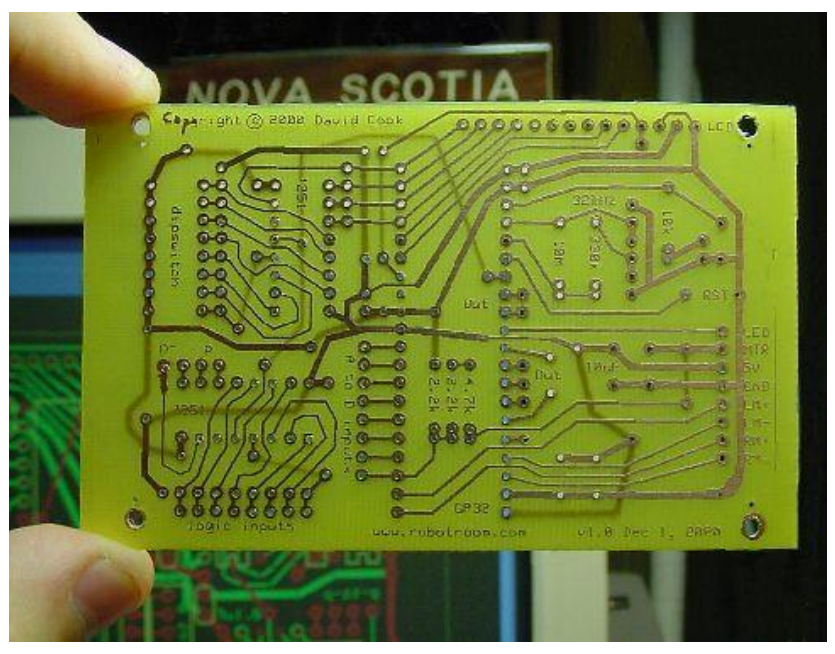

Fig.3 Designing Of PCB

PCB was initially a fully manual process done on clear Mylar sheets at a scale of usually 2 or 4 times the desired size. The schematic diagram is first converted in to a layout of components pin pads, then traces were routed to provide the required interconnections, pre-printed non-reproducing Mylar grids assisted in layout, and rub-on dry transfers of common arrangements of circuit elements (pads, contact finger integrated circuits profiles, and so on) helped standardize the layout. Traces between devices were made with self CC Cadhesive tape. The finished layout "artwork" was then photographically reproduced on the resist layers of the blank coated copper-clad boards.

\subsection{MANUFACTURING STEPS OF PCB}

\subsubsection{PCB CAM}

Manufactures never use the file directly on their equipment but always read them in their cam system. PCB cannot be manufactured progressively without cam system.

\subsubsection{PANELIZATION}

It is a procedure used to handle PCB which would otherwise be too small to process.

\subsubsection{COPPER PATTERNING}

The pattern in the manufactures PCB cam system is usually output on a photo mask.

\subsubsection{PATTERNING METHOD BY VOLUME}

It has many volumes like large volume, small volume, and hobbyist.

\subsubsection{CHEMICAL ETCHING}

It is usually done by ammonium per alphabet or ferric chloride.

\subsubsection{INNER LAYER OPTICALINSPECTION (AOI) \\ AUTOMATED}

The inner layer is given complete machine inspection before lamination process because after the lamination process the mistakes cannot be corrected.

\subsubsection{LAMINATION}

Multilayer PCB has trace layers inside the board .Lamination is done by placing the stack of materials in a press and applying pressureand heat for a period of time.

\subsubsection{DRILLING}

Holes and typically drilled with PCB board with PCB board with required diameter.

\subsubsection{PLATING AND COATING}

PCB plated with solder, tin, or gold over nickel as a resister for etching away the unneeded underlying copper.

\subsubsection{SOLDER RESIST APPLICATION}

Areas that should not per soldiered may be covered with solder resist.

It is often printed on one or both sides of the PCB.

\subsubsection{LEGEND PRINTING}

It is often printed on one or both sides of the PCB. 


\subsubsection{BARE BOARD TESTING}

It used to check whether the connections are correct or not.

\subsubsection{PROTECTION AND PACKING}

PCB intended for extreme environments often have conformal coating which is applied by dipping or spraying after the components are have soldered and finally packed

\subsection{RESISTOR}

The resistor is a two terminal electronic components designed to oppose an electronic current by producing a voltage drop between its terminals in proportion to the current that is in accordance with ohm's law

\section{Ohms law V=IR}

The resistance is equal to the voltage drop $\mathrm{v}$ across the resistor divided by the current through the resistor. resistor are characterized primarily by the resistance and the power they can dissipate other components include temperature co-efficient, noise and inductance, practical resistor can be made of resistive wire and various compounds and films and they can be integrated in to hybrid printed circuits

The size bad position of leads is relevant must be physically large enough not to overheat when dissipating their power variable resistor adjustable by changing the position

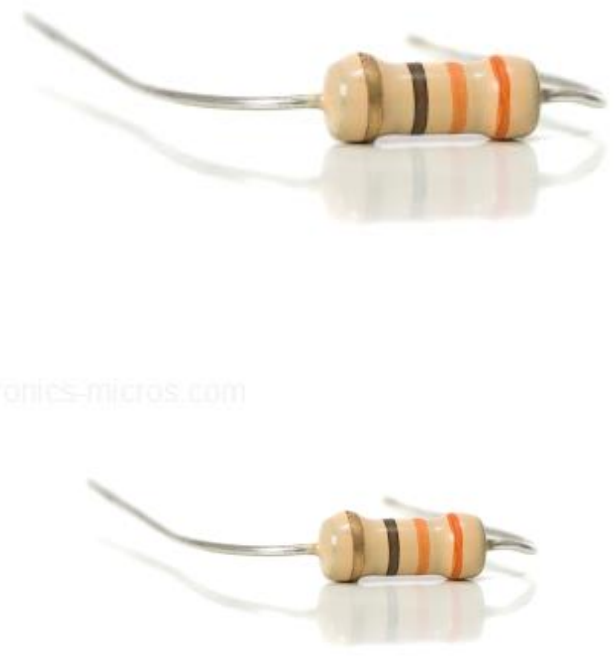

Of tapping on the resistive element and resistors with a movable tap ("potentiometer") either adjustable by the user of equipment or contained within are used.

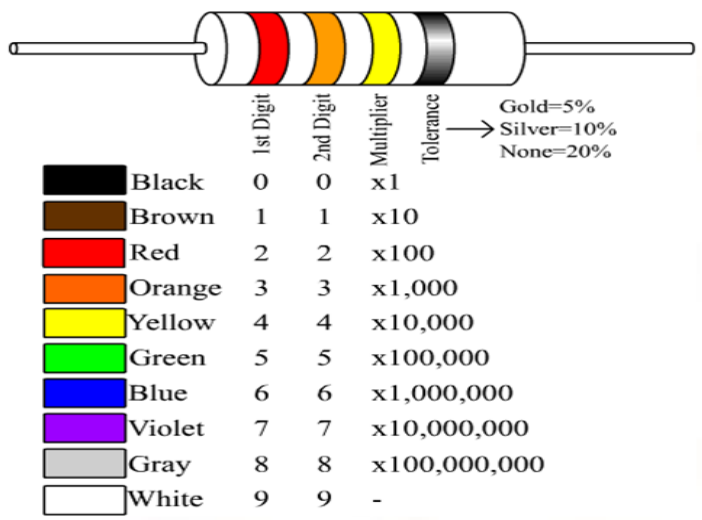

Fig 5: Colors Of The Resistors

\subsubsection{IDENTIFYING THE RESISTORS}

Separate the resistors from all other components and place them on the bench. So that the gold band indicates the resistors have a tolerance of $5 \%$.in other words they are made accurate than the older $10 \%$ types. The gold band does not concern us in this course but it does tell us which way around to hold the resistors so that the color bands can be read correctly only 10 different colors are used for all resistors

Hold the resistor so that 3 color band are to the left and the right hand band is either gold or silver. The first color gives the first digit of the resistance. The second color gives the second digit in the answer. The third color gives the number of zeros in the answer. There are only 12 resistors in each decade and they have the following first two colors.

\subsection{CAPACITOR}

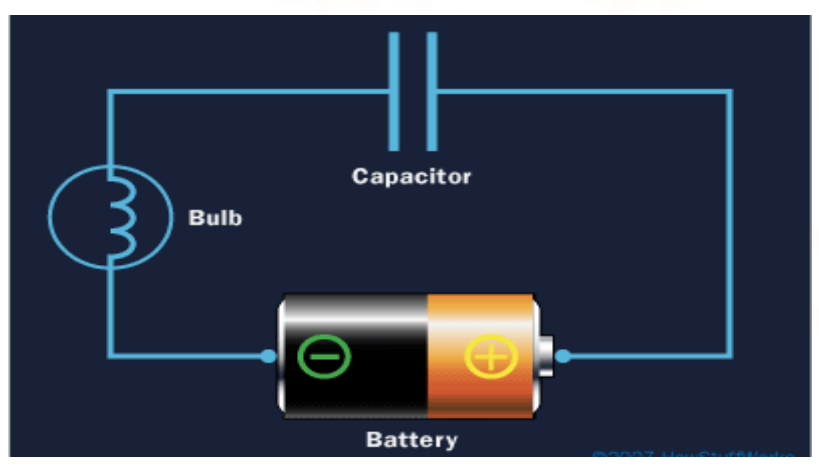


In the capacitors the plates store the energy sort like a battery the big different between the batteries and capacitors works at all voltages up to its maximum safe rating. Basically when DC voltage source is applied to the capacitor is equal to the applied voltage the current flow stops. When the current stops flowing from the power supply to the capacitor the capacitor is discharge up. After the capacitor is charge it can be discharged in like a battery.

The only problem is that the capacitor will not want to hold its charge for more than a few minutes. These present obvious problems using the capacitors as a storage devices. There are many materials make capacitors these includes the metals such as aluminum, titanium and silver is the materials used for making the capacitors.

\subsection{HOME APPLIANCES USED IN OUR PROJECT}

\subsubsection{FAN}

The coolant fan is used in our project it is controlled by the 5 volts,

\subsubsection{LIGHT}

The normal home use bulb is used it is controlled by $40,60,100 \ldots$...volts.

\section{BLOCK DIAGRAM}

\subsection{TRANSMITTER}

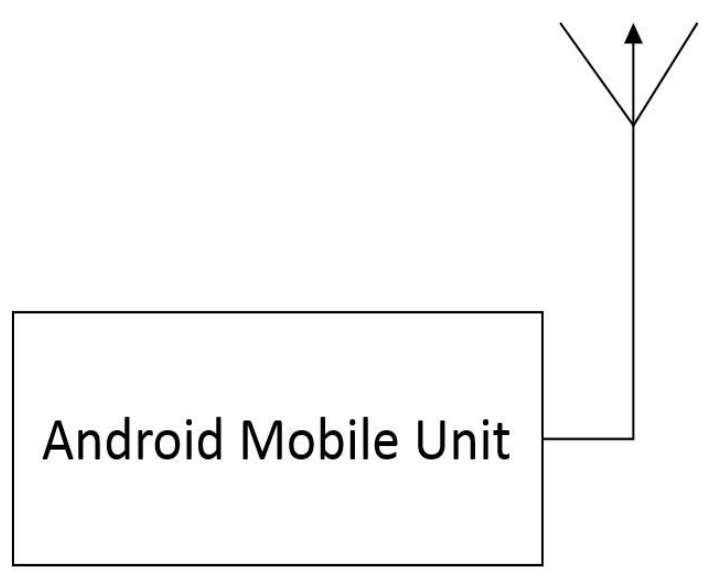

Fig 7: Transmitter

\subsection{WORKING PRINCIPAL}

The transmitter produces the short duration high power radio frequency pulses of energy that and radiated in to space by the antenna. The transmitter side have the android smart phone to transmit the signal. The transmitter is required to have the following technical and operating characteristics:

The transmitter must have the ability to generate the required mean power and the required peak power.

$>$ The rather transmitter must have a suitable radio frequency band width

The transmitter must have a high radio frequency stability to meet signal processing requirements

$>$ The transmitter must be easily modulated to meet wave from design requirements.

The transmitter must be efficient reliable and easy to maintain the life expectancy and the cost of the output device must be acceptable.

\subsection{RECEIVER}

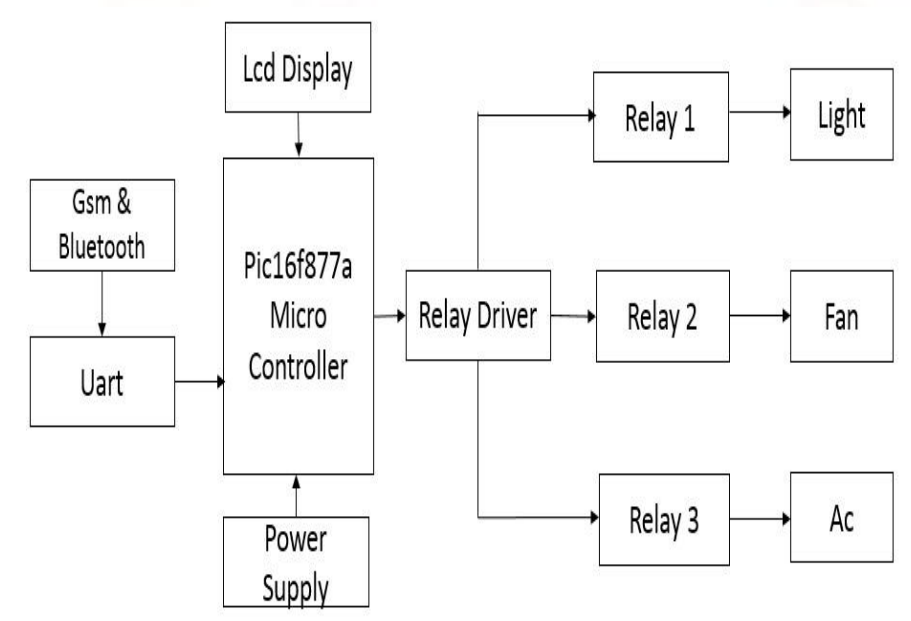

Fig 8: Receiver

\subsection{WORKING PRINCIPLE}

The receiver is the listener, reader or observer in the communication process. Another name is "Audience". In communication process the role of the receiver is believable as important as that of sender. The receiver has five important steps as follows: 
$>$ RECEIVER

$>$ UNDERSTAND

$>$ ACCEPT

$>$ USE AND

\section{GIVE FEEDBACK}

Without these steps the communication will not be successful. The sender transmitts a message and the receiver decodes a message. The receiver is the destination of the message.the task is to interpret the senders message both verbal and non verbalwith as little distortion or noise is possible .The receiver is close-mineded and unreceptive to new and different ideas . the receiver side consists of pic micro controller (PIC 16F877A),GSM (Global System For Mobile Communication),UART(Universal Asynchronous Receiver and Transmitter),power supply,bluetooth,relay driver. The signal transmitted by the android smart phone is received by the receiver.

\section{BLUETOOTH}

Bluetooth is a wireless devices used at the particular distances. it is specification IEEE (802.15.1)for the use of low power radio communications to link phones, computers and other network devices over short distance without wire

The name Bluetooth is borrow from "Haraid Bluetooth" a king in Denmark lived more than 1,000 years ago. Bluetooth technology was designed primarily to support simple wireless networking of personal consumer devices and peripheral's including all phone PDAS ,and wireless signals transmitted with Bluetooth cover short distance typically up to 30MBPS feet (10 meters).Bluetooth devices are generally communicate at less than 1MBPS

\section{CIRCUIT DIAGRAM}

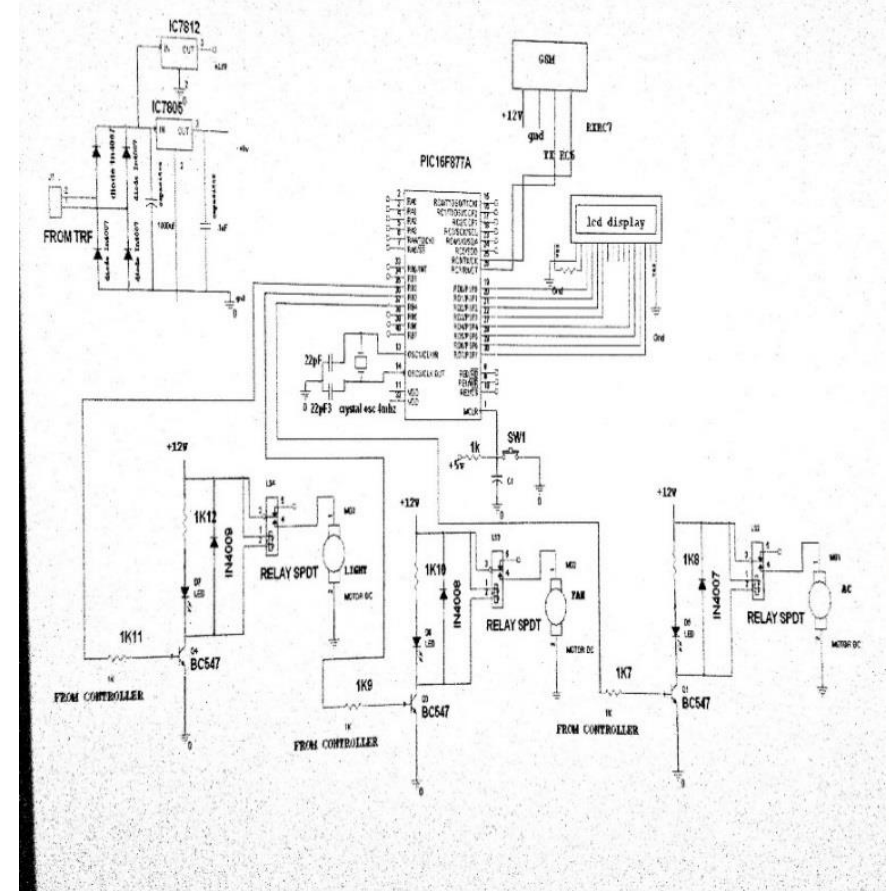

\subsection{CIRCUIT DIAGRAM EXPLANATION}

It starts from power supply section. We have the $12 \mathrm{~V}$ $\mathrm{AC}$ input from the secondary output of the transformer. This is fed to the bridge rectification section that converts AC supply in to DC supply. This is done by four IN4007, $1 \mathrm{~A}$ diodes in bridge configuration, then on the dc output of this section a large capacitor (IOOOMF, 10V) and another small $100 \mathrm{nF}$ capacitor is there to filter the DFC supply and remove all the AC components from it. This is because we are operating predigital circuitry that fails to operate on unregulated and unfiltered supply. After the DC supply is being filtered as it is unregulated is turned in to regulated 5V DC supply using LM7806 $\& 7812$ regulator.

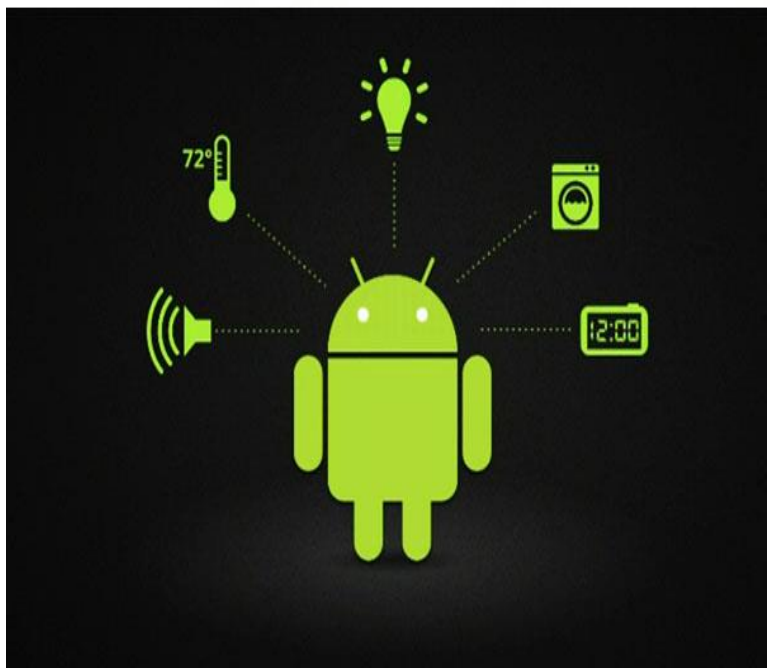


Again after that a small $100 n F$ capacitor is there to filter the regulated DC supply. A regulated 3.3volt supply is also derived from another regulator is the input is fed from the regulated $5 \mathrm{~V}$ output of the IC $7806 \& 7812$ voltage regulator. Regulated 5Volt supply is needed to drive the microcontroller, relays and all the sensors while 3.3 Volts is required by the Bluetooth

UART (universal asynchronous receiver \&transmitter) module to operate. After that the regulated supply is fed to all the sensors, relays and the microcontroller.

There are five sensors are being directly interfaced to microcontroller. To sense the light intensity we use two LDR sensor that light dependent resistors interfaced to the ADC input of the microcontroller. One pin of the LDR is connected to 330k resister and other tied to the ground. This creates the potential difference between the circuits and fed to the pin A3 and A2 of the microcontroller. To sense the temperature the two solid state semiconductor temperature sensors from analogy devices also interfaced to the ADC input of the microcontroller. The two pins are connected to the power supply.

And the third pin is the output the switches are used to sense the home appliances are on/off if it is not off the switch will be in open state. To display the messages the sensor values $16^{*} 2$ character LCD (liquid crystal display) is used in 4bit mode to port B of them. To sense the presence and the motion the PIR (PYROELECTRIC INFRARED) is used. BC 107 transistor circuit is interfaced with digital output of the microcontroller. The H-bridge circuit is used to drive the DC motor in both directions. The three transistors use the simple switch only and all the four transistors are protected by limiting the current by R1$\mathrm{R} 4$ resistors.

\subsection{EXPLANATIONAND WORKING OF ANDROID HOME AUTOMATION}

The components used in our project are android smart phone as transmitter. And the transformer the stepdown transformer is used (AC) it is (12v) 1AMPS, microcontroller (5v) DC supply it is a single layered PCB it consists of crystal oscillator KD54-000 , capacitor C5578, a GSM(global system for mobile communication) (12V)DC supply it is a double layered PCB, UART (universal asynchronous receiver and transmitter) $(12 \mathrm{~V})$ DC supply, relay driver $(12 \mathrm{v})$
DC supply it is a single layered PCB it consists of $1 \mathrm{OOOMF}$ capacitor, $22 \mathrm{MF}$ and $1 \mathrm{OMF}$ capacitor and diode IN1007, and transistor 7805GT and resistor of 330 ohms, Bluetooth (12v) it is a double layered PCB , power supply(AC to DC $5 \mathrm{~V}$ regulator and $\mathrm{DC} 12 \mathrm{v}$ is directly given to the relay driver, LCD (liquid crystal display) (12v) DC supply it is a double layered PCB and the IC is fully 5V supply are used as receivers. The signal transmitted by the android smart phone was received by the receiver side, the android 4.0version (Blue Term) is used in our project it supports the multi touch software's. The working principle of this block diagram is that the signal transmitted by the android smart phone was received by the GSM (global system for mobile communication) it covers the distance maximum up to 35 kilometers and it was connected to the universal asynchronous receiver and transmitter) UART is used for the communication between the devices and the relay is the controlling device it controls all the devices like fan, light... and finally the message was received by the android phone by using the GSM, .

\section{PIC MICRO-CONTROLLER (PIC 16F877)}

This microcontroller has only 35 single word instructions to learn. All single word cycle instructions except for program branches, which are two cycles. Operating speed in dc $20 \mathrm{MHZ}$ clock input dc-200ns instruction cycle. 2000*14 words of program memory and $128 * 8$ bytes of data memory that is RAM. It has interrupt capabilities and eightlevel deep hardware. It contains of direct, indirect and relative addressing modes. It operates on $5 \mathrm{v}$ power supply.

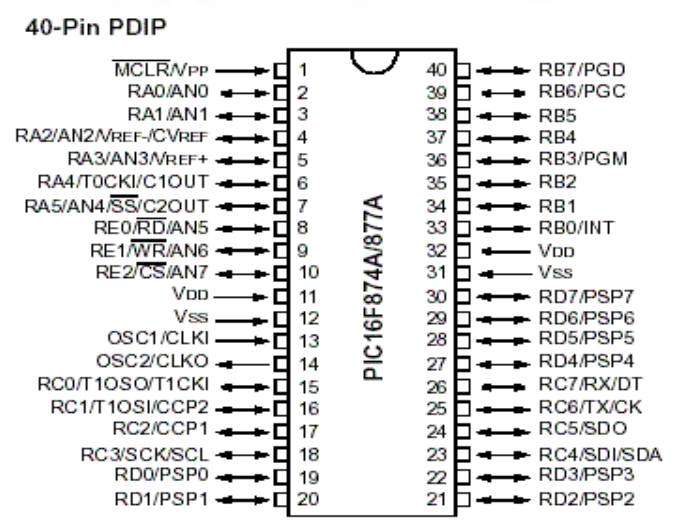

Fig.10: Pin Diagram (Pic16f877a) 
The most pins are multi-functional. For example, designator RA3/AN3/Vref+/C1IN+ for the fifth pin specifies the following functions

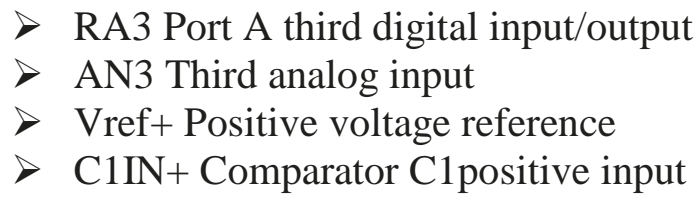

This small trick is often used because it makes the microcontroller package more compact without affecting its functionality. These various pin functions cannot be usedsimultaneously, but can be changed at any point during operation.

\section{GSM (GLOBAL SYSTEM FOR MOBILE COMMUNICATION)}

GSM (Global System for Mobile Communications, originally Group Special Mobile), is a standard set developed by the European telecommunication standard (ETSI) to describe technologies for second generation (or "20") digital cellular network. Developed as a replacement for first generation analog cellular networks, the GSM standard originally described a digital, circuit switched network optimized for duplex(communication) voice telephony. soThe standard was expanded over time to include first circuit switched data transport, then packet data transport via GPRS. Packet data transmission speeds were later increased via EDGE. The GSM standard is succeeded by the third generation ( or "30") UMTS standard developed by the 3GPP. GSM networks will evolve further as they begin to incorporate fourth generation (or " $4 \mathrm{G}$ ") LTE advanced standards. "GSM" is a trademark owned by the GSM association. The GSM Association estimates that technologies defined in the GSM standard serve $80 \%$ of the world's population, encompassing more than 5 billion people across more than 212 countries and territories, making GSM the most ubiquitous of the many standards for cellular networks.

The GSM modem is a wireless modem that works with a GSM wireless network. A wireless modem behaves like a dialup modem. The main difference between them is that a dial-up modem sends and receives data through a fixed telephone line while a wireless modem sends and receives data through radio waves

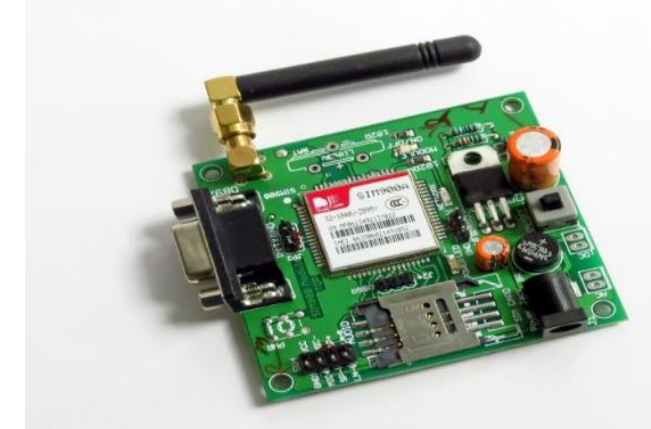

Fig 10: GSM

A GSM modem can be external device or a PC card/ Pcmcia card. Typically, an external GSM modem is connected to a computer through a serial cable or a USB cable. A GSM modem in the form of a PC card! Pcmcia card is designed for use with a laptop computer, It should be inserted in to one of the PC card/Pcmcia card slots of a laptop computer. Like GSM mobile phone, a GSM modem requires a SIM card from a wireless $\mathrm{J}$ carrier in order to operate.

As mentioned in earlier sections of this SMS tutorial, computers use AT commands to control modems. Both GSM modems and dial-up modems support a common set of standard AT commands. The GSM can use just like a dial-up modem. In addition to the standard AT commands, GSM modems support an extended set of AT commands. The extended AT commands are defined in the GSM standards. With the extended AT commands, do things like:

$>$ Reading, writing and deleting SMS messages

$>$ Sending SMS messages

$>$ Monitoring the signal strength.

$>$ Monitoring the charging status and charge level of the battery

$>$ Reading, writing and searching phone book entries,

The number of SMS messages that can be processed by a GSM modem per minute is very low only about six. to ten SMS messages per minute This GSM modem is a highly flexible plug and play GSM 850 900 / GSM 1800 / GSM 1900 modem for direct and easy integration R8232, voltage range for the power supply and audio interface make this device perfect solution for system integrators and single user. It 'also comes with license free integrated Python. Python is a powerful easy to learn programming language. Such a Python driven terminal is 5 times better and faster and 5 times cheaper than standard PLC/RTU with communication interface and external GSM f GPRS 
modem. Voice, Data/Fax, SMS, DTMF, GPRS, integrated TCP/IP stack, RTC and other features like the GSM / GPRS modules on this home page.

\subsection{GSM FEATURE}

$>$ GPRS class 10:max 85.6 KBPS(down link)

$>$ PBCCH support

$>$ Coding schemes CS1,2,3,4

$>\mathrm{CDS}$ up to $14.4 \mathrm{KBPS}$

$>$ USSD

\section{RELAY}

Relays are used throughout the most of appliances that comes in different sizes for suitable appliances. And also it is used as remote control switches. A typical vehicle can have 20 relays or more. It is purely for switching purpose. All relays operate using the same basic principle. Our example will use a commonly used 4-pin relays. It has two circuits. Control circuit has a small control coil and load circuit has a switch. The whole coil controls the operation of switch. The coil produces a magnetic field. Relays are either normally closed or open switched. When the current flows through the coil, the magnetic field will be produced at the coil, hence the open circuit will be closed and current flows through the relay. At this time relay switches and power source goes to the load. The magnetic field attracts the upper contact arm and pulls it down, closing the contacts and allowing power from the power source to go to the load.

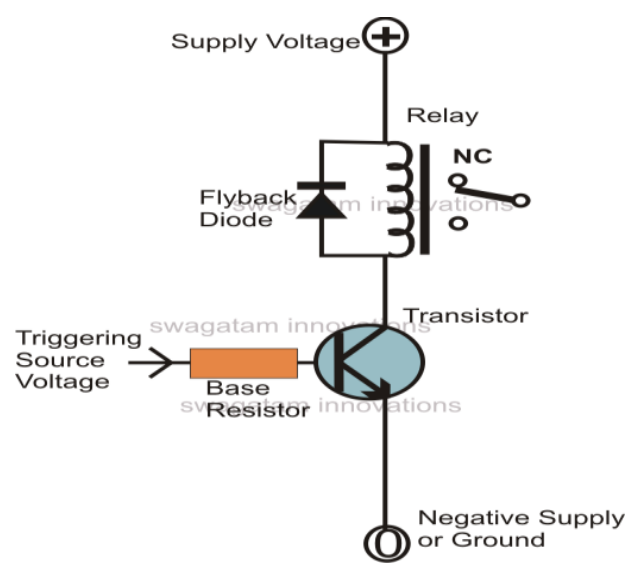

Fig 11: Relay Driver Switch

\section{POWER SUPPLY}

A power supply is a device that supplies electrical power to one or more electric loads or appliances. The term is most commonly applied to devices that convert one form of electrical energy to another, though it may also refer to devices that convert another form of energy to electrical energy. Low voltage DC power supplies are commonly integrated with their loads in devices such as computers and household electronics. Rectifier is used to convert alternating voltage into pulsating direct voltage. A small remaining unwanted alternating voltage component at mains or twice mains power frequency depending upon whether half or full wave rectification is used ripple is unavoidably Super imposed on the direct output voltage. For purpose such as charging batteries the ripple is not a problem, and the simplestUNregulated mains-powered DC power supply circuit consists of transformer driving a single diode in series with a resistor.

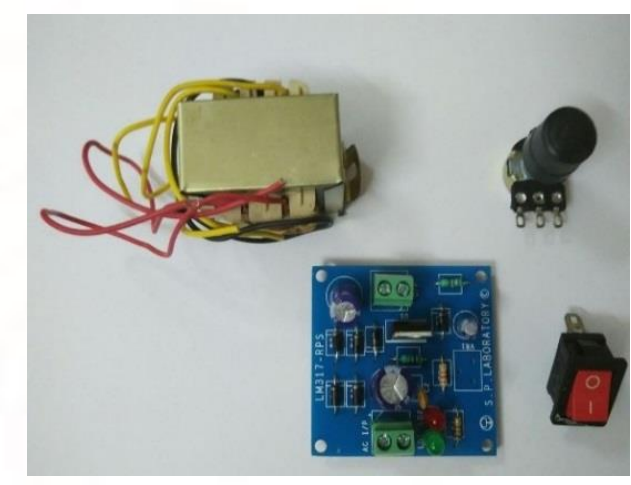

Fig.12: Power Supply

\section{LCD display}

A LCD display is thin and flat electronic visual display used to show the info to the users. Here we used this display inside the vehicle to show the details that from mobile phone caller. It is used instead of mobile phone display. We used $16 * 2$ character LCD display. They are usually more compact, lightweight, portable, less expensive and easier to visible on the eyes.

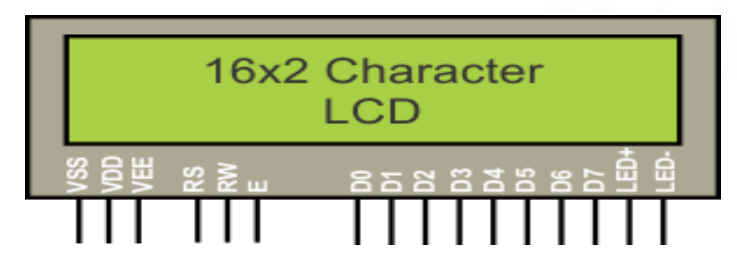

Fig13: 16*2 LCD Display 
The receiver section contains 8051 microcontroller, relay driver circuit and ignition system.

\section{LED (LIGHT EMMITING DIODE)}

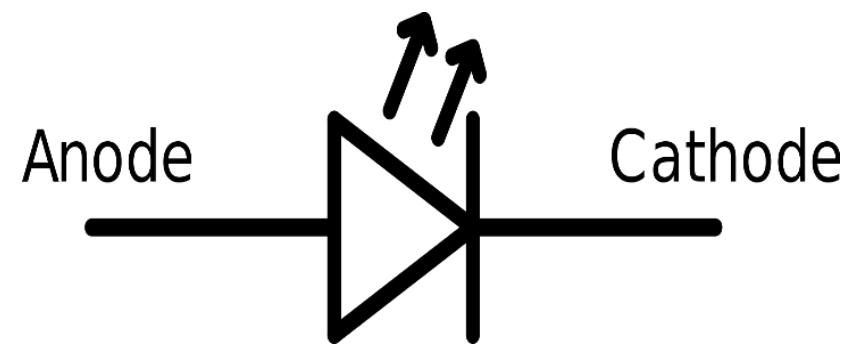

Fig 14: Symbol of LED

A light-emitting diode (LED) is a twolead semiconductor light source. It is a P-N Junction Diode that emits light when activated. When a suitable voltage is applied to the leads, electrons are able to recombine with electron holes within the device, releasing energy in the form of photons. This effect is called electroluminescence, and the color of the light (corresponding to the energy of the photon) is determined by the energy band gap of the semiconductor. LEDs are typically small (less than $1 \mathrm{~mm}^{2}$ ) and integrated optical components may be used to shape the radiation pattern

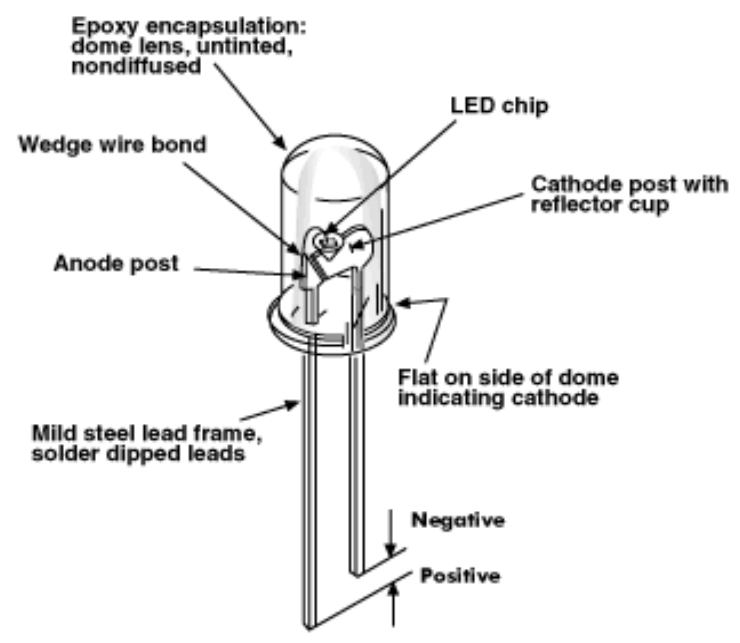

Fig.15

\section{ADVANDAGE}

$>$ The main advantage is that it increase the efficiency

$>$ It covers the main areas.

$>$ It reacts for the changes intelligently like turning the lights when we enter the room
It can also tie in home cinema system so a single remote can turn on a projector and start media source

$>$ It reduces energy consumption

$>$ It increases the security and makes the home more comfortable.

$>$ It saves the money and time.

\section{APPLICATIONS}

$>$ It uses in security systems.

$>$ It uses in controlling the scheduled tasks.

$>$ It is used in any industries of heating, ventilating air conditioning, which Includes temperature control and humidity control.

$>$ It is used in water monitoring and onloff the electrical bulbs around the home.

$>$ It is used in independence security for old peoples.

$>$ It is used in web applications

$>$ It is used in robotics.

$>$ It is used in for communication with multiple rooms.

$>$ It is used in video and communications.

$>$ It is used in kitchen appliance.

$>$ It is used in testing and certifications.

\section{FEATURE ENHANCEMENT}

$>$ It will become a more luxury business

$>$ Going to be generic.

$>$ It will have increasing role in IP networks.

$>$ Read just business models

$>$ New kind of installer.

\section{CONCLUTION}

In our everyday life we integrated with mobile devices. The home appliance can be controlled through android mobile device. Our project is developed a Bluetooth on mobile device. The mobile device and security system get communicate with this Bluetooth The status is updated on android mobile device through Bluetooth and displayed an LCD. This is essential because of the wide range of technical knowledge that home users have. Our project becomes a prominent one. So it is useful for everyone in the world. 


\section{REFERENCE}

1) AlfredJugel , Hendrik Jan Hamann Kurzawa, Ulrich schoen, and Christian, (1998),"convergence between public switching and the internet",ieee communication Magazine, vol .35,issue 1 ,pp. 50-65Ben lee,

2) Daewong Kim , Dongman lee ,hyun , saehoonKang J, and Younghee lee, (2007),"A Semantic Services Discovery Network For Large - scale Ubiquitous Computing “, ETRI journal, vol 29, no.5,pp 545-558.

3) Bruce NordmanChamaraGunarathe, and Ken Christensen, (2005),'Managing energy Consumption Costs in Desktop PCs and LAN switches with proxing, Split TCP connections, and Scaling Of link speed :,International Journal of Network Management,pp 297-310.

4) Chang-sic choi, Kwangroh Prak, sung -II nam, Wan-Ki Park, and Youn-Kwaejeong, (2006),"An implementation of FTTH based Home Gate ways Supporting Various Services: IEEE Transactions on consumer Electronics, vol.52, No.1, pp110-115.

5) Kerpezetal .k, (2006),'’IPTV Service Assurance", IEEE Communications Magazine, vol.44, no.9, pp.166-72. 